\title{
The Poisson-Transmuted Janardan Distribution for Modelling Count Data
}

\author{
Winai Bodhisuwan ${ }^{1}$ and Sirinapa Aryuyuen, ${ }^{2, *}$ \\ ${ }^{I}$ Department of Statistics, Faculty of Science, Kasetsart University, Bangkok 10900, Thailand \\ ${ }^{2}$ Department of Mathematics and Computer Science, Faculty of Science and Technology, \\ Rajamangala University of Technology Thanyaburi, Pathum Thani 12110, Thailand
}

("Corresponding author's e-mail: sirinapa_a@rmutt.ac.th)

Received: 10 October 2020, Revised: 17 May 2021, Accepted: 27 May 2021

\begin{abstract}
In this paper, we introduce a new mixed Poisson distribution, called the Poisson-transmuted Janardan distribution. The Poisson-Janardan and Poisson-Lindley distributions are sub-model of the proposed distribution. Some mathematical properties of the proposed distribution, including the moments, moment generating function, probability generating function and generation of a Poisson-transmuted Janardan random variable, are presented. The parameter estimation is discussed based on the method of moments and the maximum likelihood estimation. In addition, we illustrated the application of the proposed distribution by fitting with 4 real data sets and comparing it with some other distributions based on the Kolmogorov-Smirnov test for criteria.
\end{abstract}

Keywords: Mixed Poisson distribution, Poisson-transmuted Janardan distribution, Count data, Overdispersion, Probability function

\section{Introduction}

Count data are used to describe many phenomena in different fields from insurance and economics to biometrics and the social sciences. Count data analysis can use traditional models such as the Poisson distribution to describe the data if the variance and mean are equal. However, in practice, it has often been found that count data exhibit over-dispersion (variance greater than mean). Hence, there is a demand to modify the Poisson model when such problems are encountered. In the last 2 decades, many researchers have developed new distributions to count data analysis. One such method has been wide that has been widely used for observed modeling situations is a mixture of distributions [1,2].

A new mixed Poisson distribution is a mixture of distributions for the count data. A traditional mixed Poisson distribution where the mean of the Poisson variable is distributed as a gamma random variable is the Poisson-gamma (or the negative binomial) distribution, derived by [3]. It has become an increasingly popular alternative distribution to the Poisson distribution. However, the Poisson-gamma distribution may not be appropriate for some over-dispersed count data. Some well-known mixed Poisson distributions are the Poisson-Lindley by [4], the Poisson-inverse Gaussian by [5], the Poisson-Pareto by [6], the Poisson-Lomax by [7], generalized Poisson-Lindley by [8], the Poisson-weight exponential by [9], the Poisson-Janardan by [10], the Poisson-Lindley beta by [11], the Poisson-generalized Lindley by [12], etc. Recently, the Poisson-transmuted exponential was introduced by [1]. The existing mixed Poisson distributions can be used for improving count data modeling.

In this paper, a new mixed Poisson distribution is obtained by mixing the Poisson distribution and the transmuted Janardan (TJ) distribution. The TJ distribution was proposed by [13], which is a continuous distribution. It has various structural properties that are the explicit expressions for the reliability and hazard rate functions, order statistics, the moments and the moment the generating function. The TJ distribution is suggested for several applications in the modeling of real data. We describe the theoretical development of the mixed Poisson distribution. A new mixed Poisson distribution, so-called the Poisson-transmuted Janardan distribution, is proposed. In addition, some properties of the proposed distribution are shown, such as reliability and hazard rate functions, order statistics, moments and moment generating function. The different methods of parameter estimation are derived, i.e., the method of moments and the maximum likelihood estimation. The simulation study of the 
parameter estimation based on the MME and MLE is shown. Moreover, an application of the proposed distribution is illustrated. Finally, some discussion and conclusions are included.

\section{Methods}

Let $\mathrm{X}$ be a random variable which is distributed the Poisson distribution with a parameter $\lambda$. The probability mass function $(\mathrm{pmf})$ of $\mathrm{X}, \mathrm{g}_{\text {Pois }}(\mathrm{x})$, is;

$\mathrm{g}_{\text {Pois }}(\mathrm{x})=\frac{\mathrm{e}^{-\lambda} \lambda^{\mathrm{x}}}{\mathrm{x} !} ; \mathrm{x}=0,1,2, \ldots, \lambda>0$

A new mixed Poisson distribution arises from the Poisson distribution when its parameter $\lambda$ follows the TJ distribution. The probability density function (pdf) of the TJ distribution [13] is;

$$
\mathrm{g}_{\mathrm{TJ}}(\lambda)=\frac{\theta^{2}(\alpha \lambda+1) \mathrm{e}^{-2 \theta \lambda / \alpha}}{\alpha\left(\alpha^{2}+\theta\right)^{2}}\left[2 \beta\left(\alpha^{2}+\theta+\alpha \theta \lambda\right)-(\beta-1)\left(\alpha^{2}+\theta\right) \mathrm{e}^{\theta \lambda / \alpha}\right],
$$

where $\alpha, \theta>0$ and $-1 \leq \beta \leq 1$. For $\beta=0$ the TJ distribution reduces to the Janardan distribution, as proposed by [14]. The cumulative density function (cdf) of the Janardan distribution, $G_{J}(\lambda)$, is;

$$
\mathrm{G}_{\mathrm{J}}(\lambda)=1-\frac{\alpha\left(\alpha^{2}+\theta\right)+\alpha^{2} \theta \lambda}{\alpha\left(\alpha^{2}+\theta\right)} \mathrm{e}^{-\frac{\theta \lambda}{\alpha}}
$$

and the inverse cdf is;

$$
\mathrm{G}_{\mathrm{J}}^{-1}(\mathrm{v})=\frac{\alpha}{\theta}\left\{-\frac{\alpha^{2}+\theta}{\alpha^{2}}-\mathrm{W}_{-1}\left[-\frac{\alpha^{2}+\theta}{\alpha^{2}}(1-\mathrm{v}) \mathrm{e}^{-\frac{\alpha^{2}+\theta}{\alpha^{2}}}\right]\right\}
$$

where $\mathrm{v}$ is a value of a uniform random variable on $[0,1]$, and $\mathrm{W}_{-1}($.) denotes the negative branch of the Lambert $\mathrm{W}$ function [15], i.e., $\mathrm{W}(\mathrm{z})=\mathrm{e}^{\mathrm{W}(\mathrm{z})}=\mathrm{z}$, where $\mathrm{z}$ is a complex number. We apply the Lambert $\mathbf{W}$ function in $\mathrm{R}[16]$ to solve the $\mathrm{W}_{-1}$ (.). Since the TJ distribution is the quadratic rank transmutation. Thus, we obtained the inverse cdf of the TJ distribution by using the cdf of the quadratic rank transmutation [17], that is;

$$
\mathrm{G}_{\mathrm{T} \lambda}(\lambda)=(1+\beta) \mathrm{G}_{\lambda}(\lambda)-\beta\left[\mathrm{G}_{\lambda}(\lambda)\right]^{2} ;-1 \leq \beta \leq 1,
$$

where $G_{\lambda}(\lambda)$ is the cdf of the baseline distribution. Then the inverse $G_{T \lambda}(\lambda)$ is

$$
\mathrm{G}_{\mathrm{T} \lambda}^{-1}(\mathrm{u})=\mathrm{G}_{\lambda}^{-1}\left\{\frac{1}{2 \beta}\left[1+\beta-\sqrt{(\beta+1)^{2}-4 \beta \mathrm{u}}\right]\right\}
$$

where $u$ is a value of a uniform random variable on $[0,1]$. When $G_{\lambda}(\lambda)$ is the cdf of the Janardan distribution as in eq. (3), we have the inverse cdf of the TJ distribution as;

$$
\mathrm{G}_{\mathrm{TJ}}^{-1}(\mathrm{u})=\mathrm{G}_{\mathrm{J}}^{-1}\left\{\frac{1}{2 \beta}\left[1+\beta-\sqrt{(\beta+1)^{2}-4 \beta \mathrm{u}}\right]\right\}
$$




$$
=\frac{\alpha}{\theta}\left\{-\frac{\alpha^{2}+\theta}{\alpha^{2}}-\mathrm{W}_{-1}\left\{-\frac{\alpha^{2}+\theta}{\alpha^{2}} \mathrm{e}^{-\frac{\alpha^{2}+\theta}{\alpha^{2}}}\left[1-\frac{1+\beta-\sqrt{(\beta+1)^{2}-4 \beta \mathrm{u}}}{2 \beta}\right]\right\}\right\} .
$$

\section{Results and discussion}

\section{A new mixed Poisson distribution}

By mixing the Poisson and TJ distributions in Definition 1, we obtained a new mixed Poisson distribution, and its pmf is in Theorem 1.

Definition 1 Let $X$ be a random variable of the Poisson-transmuted Janardan (PTJ) distribution, will be denoted by $\mathrm{X} \sim \operatorname{PTJ}(\alpha, \theta, \beta)$, when $\mathrm{X} \mid \lambda$ has distributed the Poisson (Pois) distribution with a positive parameter $\lambda$. Where the parameter $\lambda$ has distributed the TJ distribution with the parameters $\alpha>0$, $\theta>0$ and $-1 \leq \beta \leq 1$, i.e., $\mathrm{X} \mid \lambda \sim \operatorname{Pois}(\lambda)$ and $\lambda \sim \mathrm{TJ}(\alpha, \theta, \beta)$.

Theorem 1 Let $\mathrm{X} \sim \operatorname{PTJ}(\alpha, \theta, \beta)$ then the pmf of $\mathrm{X}$ is;

$$
\begin{aligned}
\mathrm{f}_{\mathrm{PTJ}}(\mathrm{x}) & =\frac{\theta^{2} \alpha^{\mathrm{x}}}{\left(\alpha^{2}+\theta\right)^{2}}\left\{\frac{2 \beta}{(\alpha+2 \theta)^{\mathrm{x}+1}}\left[\frac{\alpha^{2} \theta(\mathrm{x}+1)}{\alpha+2 \theta}\left(1+\frac{\alpha^{2}(\mathrm{x}+2)}{\alpha+2 \theta}\right)+\left(\alpha^{2}+\theta\right)\left(1+\frac{\alpha^{2}(\mathrm{x}+1)}{\alpha+2 \theta}\right)\right]\right. \\
& \left.-\frac{(\beta-1)\left(\alpha^{2}+\theta\right)}{(\alpha+\theta)^{\mathrm{x}+1}}\left(1+\frac{\alpha^{2}(\mathrm{x}+1)}{\alpha+\theta}\right)\right\},
\end{aligned}
$$

where $\mathrm{x}=0,1,2, \ldots$ and the parameters $\alpha, \theta>0$ and $-1 \leq \beta \leq 1$.

Proof Since $\mathrm{X} \mid \lambda \sim \operatorname{Pois}(\lambda)$ and $\lambda \sim \mathrm{TJ}(\alpha, \theta, \beta)$, the pmf of the PTJ distribution will be obtained utilization of;

$$
\mathrm{f}_{\text {PTJ }}(\mathrm{x})=\int_{0}^{\infty} \mathrm{g}_{\text {Pois }}(\mathrm{x} \mid \lambda) \mathrm{g}_{\mathrm{TJ}}(\lambda) \mathrm{d} \lambda
$$

By substituting (1) and (2) into (7), we derive the PTJ pmf;

$$
\begin{aligned}
\mathrm{f}_{\mathrm{PTJ}}(\mathrm{x}) & =\int_{0}^{\infty} \frac{\mathrm{e}^{-\lambda} \lambda^{\mathrm{x}}}{\mathrm{x} !} \frac{\theta^{2}(\alpha \lambda+1) \mathrm{e}^{-2 \theta \lambda / \alpha}}{\alpha\left(\alpha^{2}+\theta\right)^{2}}\left[2 \beta\left(\alpha^{2}+\theta+\alpha \theta \lambda\right)-(\beta-1)\left(\alpha^{2}+\theta\right) \mathrm{e}^{\theta \lambda / \alpha}\right] \mathrm{d} \lambda \\
& =\frac{\theta^{2}}{\alpha\left(\alpha^{2}+\theta\right)^{2} \Gamma(\mathrm{x}+1)}\left[2 \beta \int_{0}^{\infty}\left(\alpha \lambda^{\mathrm{x}+1}+\lambda^{\mathrm{x}}\right)\left(\alpha^{2}+\theta+\alpha \theta \lambda\right) \mathrm{e}^{-\left(\frac{\alpha+2 \theta}{\alpha}\right) \lambda} \mathrm{d} \lambda\right. \\
& \left.-(\beta-1)\left(\alpha^{2}+\theta\right) \int_{0}^{\infty}\left(\alpha \lambda^{\mathrm{x}+1}+\lambda^{\mathrm{x}}\right) \mathrm{e}^{-\left(\frac{\alpha+\theta}{\alpha}\right) \lambda} \mathrm{d} \lambda\right] .
\end{aligned}
$$

Let $\mathrm{u}=\left(\frac{\alpha+2 \theta}{\alpha}\right) \lambda$ and $\mathrm{v}=\left(\frac{\alpha+\theta}{\alpha}\right) \lambda$, we have $\mathrm{d} \lambda=\left(\frac{\alpha}{\alpha+2 \theta}\right) \mathrm{du}$ and $\mathrm{d} \lambda=\left(\frac{\alpha}{\alpha+\theta}\right) \mathrm{dv}$, respectively. The corresponding pmf in (8) is;

$$
\begin{aligned}
\mathrm{f}_{\mathrm{PTJ}}(\mathrm{x}) & =\frac{\theta^{2}}{\alpha\left(\alpha^{2}+\theta\right)^{2} \Gamma(\mathrm{x}+1)}\left[2 \beta \left[\alpha\left(\alpha^{2}+\theta\right)\left(\frac{\alpha}{\alpha+2 \theta}\right)^{\mathrm{x}+2} \Gamma(\mathrm{x}+2)+\alpha^{2} \theta\left(\frac{\alpha}{\alpha+2 \theta}\right)^{\mathrm{x}+3} \Gamma(\mathrm{x}+3)\right.\right. \\
& \left.+\left(\alpha^{2}+\theta\right)\left(\frac{\alpha}{\alpha+2 \theta}\right)^{\mathrm{x}+1} \Gamma(\mathrm{x}+1)+\alpha \theta\left(\frac{\alpha}{\alpha+2 \theta}\right)^{\mathrm{x}+2} \Gamma(\mathrm{x}+2)\right]
\end{aligned}
$$




$$
\left.-(\beta-1)\left(\alpha^{2}+\theta\right)\left[\alpha\left(\frac{\alpha}{\alpha+\theta}\right)^{x+2} \Gamma(x+2)+\left(\frac{\alpha}{\alpha+\theta}\right)^{x+1} \Gamma(x+1)\right]\right\} .
$$

Finally, we obtain the pmf of the PTJ distribution as in (6). The pmf of X satisfies the following properties: (i) $\mathrm{f}_{\mathrm{PTJ}}(\mathrm{x}) \geq 0$ and (ii) $\sum_{\mathrm{x}} \mathrm{f}_{\mathrm{PTJ}}(\mathrm{x})=1$ for all $\mathrm{x}$.

The pmf behavior of the proposed distribution is shown in Figure 1. The PTJ pmf has a decreasing function for $\alpha<1$, see Figures 1(a) and 1(b). If $\alpha \geq 1$ then the pmf of the PTJ distribution has a unimodal distribution, see Figures 1(c) and 1(d). The PTJ distribution has 2 sub-model as follows.

Corollary 1 Let $\mathrm{X} \sim \operatorname{PTJ}(\alpha, \theta, \beta)$, if $\beta=0$ then PTJ distribution reduce to the Poisson-Janardan (PJ) distribution, which the PJ distribution is introduced by [10]. The pmf of the PJ distribution is;

$f_{P J}(x)=\left(\frac{\theta}{\alpha+\theta}\right)^{2}\left(\frac{\alpha}{\alpha+\theta}\right)^{x}\left[1+\frac{\alpha(\alpha x+1)}{\alpha^{2}+\theta}\right] ; x=0,1,2, \ldots, \alpha>0, \theta>0$.

Corollary 2 Let $\mathrm{X} \sim \operatorname{PTJ}(\alpha, \theta, \beta)$, if $\beta=0$ and $\alpha=1$ then the PTJ distribution reduces to the PoissonLindley (PL) distribution, which the PL distribution is introduced by [4]. The pmf of the PL distribution is;

$f_{P L}(x)=\frac{\theta^{2}(2+\theta+x)}{(1+\theta)^{x+3}} ; x=0,1,2, \ldots, \theta>0$.

(a) $\alpha=0.05, \theta=0.05, \beta=0.75$

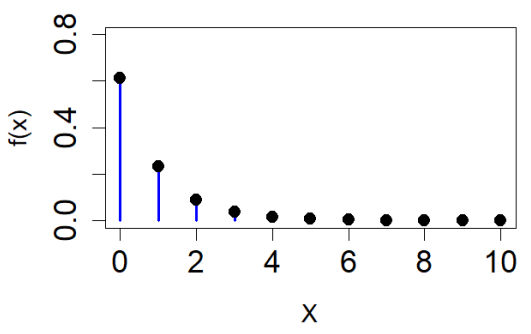

(c) $\alpha=1.5, \theta=0.5, \beta=0.2$

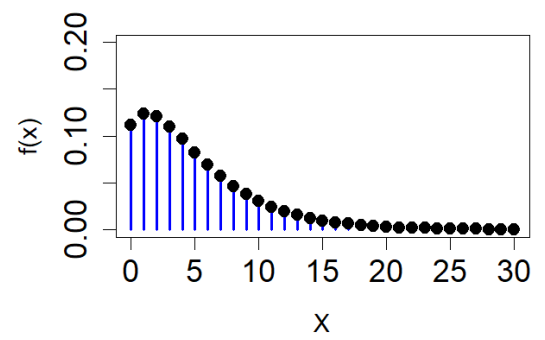

(b) $\alpha=0.15, \theta=0.15, \beta=0.5$

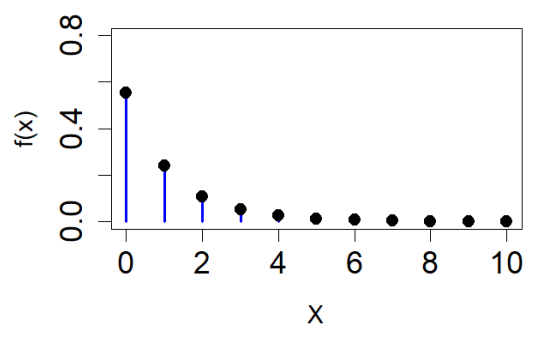

(d) $\alpha=3, \theta=0.5, \beta=0.7$

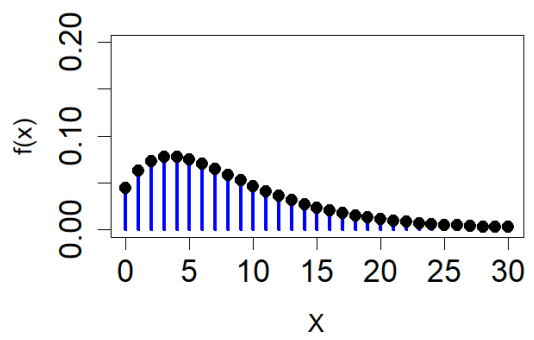

Figure 1 The PJT pmf plots with some specified value of parameters $\alpha, \theta$ and $\beta$.

\section{Properties of the PTJ distribution}

Some properties of the proposed distribution including, the moments, moment generating function, probability generating function and the generating of a PTJ random variable, are introduced.

Proposition 1 If $\mathrm{X} \sim \operatorname{PTJ}(\alpha, \theta, \beta)$, then the $\mathrm{k}$ th factorial moment of $\mathrm{X}$ is; 


$$
\begin{aligned}
\mu_{\mathrm{k}}^{\prime} & =\frac{1}{\left(\alpha^{2}+\theta\right)^{2}}\left(\frac{\alpha}{\theta}\right)^{\mathrm{k}}\left\{\left(\alpha^{2}+\theta\right)\left[\alpha^{2} \Gamma(\mathrm{k}+2)+\theta \Gamma(\mathrm{k}+1)\right]-\frac{\beta}{4}\left[\alpha^{4}\left(4 \Gamma(\mathrm{k}+2)-\frac{\Gamma(\mathrm{k}+3)+2 \Gamma(\mathrm{k}+2)}{2^{\mathrm{k}}}\right)\right.\right. \\
& \left.\left.+4 \theta^{2} \Gamma(\mathrm{k}+1)\left(1-\frac{1}{2^{\mathrm{k}}}\right)+4 \alpha^{2} \theta\left(\Gamma(\mathrm{k}+2)+\Gamma(\mathrm{k}+1)-\frac{\Gamma(\mathrm{k}+2)+\Gamma(\mathrm{k}+1)}{2^{\mathrm{k}}}\right)\right]\right\},
\end{aligned}
$$

where $\mathrm{k}=1,2,3, \ldots$, and the parameters $\alpha, \theta>0$ and $-1 \leq \beta \leq 1$.

Proof The kth moment about the origin of the PTJ distribution can be obtained as;

$$
\mu_{\mathrm{k}}^{\prime}=\mathrm{E}\left[\mathrm{E}\left(\mathrm{X}^{\mathrm{k}} \mid \lambda\right)\right]=\int_{0}^{\infty}\left[\sum_{\mathrm{x}=0}^{\infty} \frac{\mathrm{x}^{\mathrm{k}} \mathrm{e}^{-\lambda} \lambda^{\mathrm{x}}}{\Gamma(\mathrm{x}+1)}\right] \frac{\theta^{2}(\alpha \lambda+1) \mathrm{e}^{-\frac{2 \theta \lambda}{\alpha}}}{\alpha\left(\alpha^{2}+\theta\right)^{2}}\left[2 \beta\left(\alpha^{2}+\theta+\alpha \theta \lambda\right)-(\beta-1)\left(\alpha^{2}+\theta\right) \mathrm{e}^{\frac{\theta \lambda}{\alpha}}\right] \mathrm{d} \lambda,
$$

since $\sum_{\mathrm{x}=0}^{\infty} \frac{\mathrm{x}^{\mathrm{k}} \mathrm{e}^{-\lambda} \lambda^{\mathrm{x}}}{\Gamma(\mathrm{x}+1)}=\lambda^{\mathrm{k}}$, we obtain;

$$
\begin{aligned}
\mu_{\mathrm{k}}^{\prime}= & \frac{\theta^{2}}{\alpha\left(\alpha^{2}+\theta\right)^{2}}\left\{2 \beta\left[\left(\alpha^{2}+\theta\right) \int_{0}^{\infty}\left(\alpha \lambda^{\mathrm{k}+1}+\lambda^{\mathrm{k}}\right) \mathrm{e}^{-\frac{2 \theta \lambda}{\alpha}} \mathrm{d} \lambda+\alpha \theta \int_{0}^{\infty}\left(\alpha \lambda^{\mathrm{k}+2}+\lambda^{\mathrm{k}+1}\right) \mathrm{e}^{-\frac{2 \theta \lambda}{\alpha}} \mathrm{d} \lambda\right]\right. \\
& \left.-(\beta-1)\left(\alpha^{2}+\theta\right) \int_{0}^{\infty}\left(\alpha \lambda^{\mathrm{k}+1}+\lambda^{\mathrm{k}}\right) \mathrm{e}^{-\frac{\theta \lambda}{\alpha}} \mathrm{d} \lambda\right\} \\
= & \frac{\theta^{2}}{\alpha\left(\alpha^{2}+\theta\right)^{2}}\left\{2 \beta \left[\alpha^{2} \theta\left(\frac{\alpha}{2 \theta}\right)^{\mathrm{k}+3} \Gamma(\mathrm{k}+3)+\alpha\left(\alpha^{2}+2 \theta\right)\left(\frac{\alpha}{2 \theta}\right)^{\mathrm{k}+2} \Gamma(\mathrm{k}+2)+\left(\alpha^{2}+\theta\right) \Gamma(\mathrm{k}+1)\right.\right. \\
& \left.\left.\times\left(\frac{\alpha}{2 \theta}\right)^{\mathrm{k}+1}\right]-(\beta-1)\left(\alpha^{2}+\theta\right)\left[\alpha\left(\frac{\alpha}{\theta}\right)^{\mathrm{k}+2} \Gamma(\mathrm{k}+2)+\left(\frac{\alpha}{\theta}\right)^{\mathrm{k}+1} \Gamma(\mathrm{k}+1)\right]\right\} .
\end{aligned}
$$

Finally, the $\mathrm{k}$ th moment about the origin of the PTJ distribution is expressed as in (11). By taking $\mathrm{k}=1,2,3$ and 4 in (11), the first 4 factorial moments about the origin of the PTJ distribution can be obtained;

$$
\begin{aligned}
& \mu_{1}^{\prime}=\frac{1}{\left(\alpha^{2}+\theta\right)^{2}}\left(\frac{\alpha}{\theta}\right)\left[\left(\alpha^{2}+\theta\right)\left(2 \alpha^{2}+\theta\right)-\frac{\beta}{4}\left(3 \alpha^{4}+2 \theta^{2}+6 \alpha^{2} \theta\right)\right], \\
& \mu_{2}^{\prime}=\frac{1}{\left(\alpha^{2}+\theta\right)^{2}}\left(\frac{\alpha}{\theta}\right)^{2}\left[\left(\alpha^{2}+\theta\right)\left(6 \alpha^{2}+2 \theta\right)-\frac{\beta}{4}\left(15 \alpha^{4}+6 \theta^{2}+24 \alpha^{2} \theta\right)\right], \\
& \mu_{3}^{\prime}=\frac{1}{\left(\alpha^{2}+\theta\right)^{2}}\left(\frac{\alpha}{\theta}\right)^{3}\left[\left(\alpha^{2}+\theta\right)\left(24 \alpha^{2}+6 \theta\right)-\frac{\beta}{4}\left(75 \alpha^{4}+21 \theta^{2}+105 \alpha^{2} \theta\right)\right], \\
& \mu_{4}^{\prime}=\frac{1}{\left(\alpha^{2}+\theta\right)^{2}}\left(\frac{\alpha}{\theta}\right)^{4}\left[\left(\alpha^{2}+\theta\right)\left(120 \alpha^{2}+24 \theta\right)-\frac{\beta}{4}\left(420 \alpha^{4}+90 \theta^{2}+540 \alpha^{2} \theta\right)\right] .
\end{aligned}
$$

From the first 4 factorial moments of the PTJ distribution, the mean, variance, index of dispersion (ID), skewness (Sk) and kurtosis (Ku) of $\mathrm{X}$ are respectively given by; 


$$
\begin{aligned}
& \mathrm{E}(\mathrm{X})=\frac{\alpha}{4 \theta\left(\alpha^{2}+\theta\right)^{2}}\left[4\left(\alpha^{2}+\theta\right)\left(2 \alpha^{2}+\theta\right)-\beta\left(3 \alpha^{4}+2 \theta^{2}+6 \alpha^{2} \theta\right)\right] \\
& \mathrm{V}(\mathrm{X})=\frac{\alpha^{2}}{\theta^{2}\left(\alpha^{2}+\theta\right)^{2}}\left\{\left[2\left(\alpha^{2}+\theta\right)\left(3 \alpha^{2}+\theta\right)-\frac{3 \beta}{4}\left(5 \alpha^{4}+2 \theta^{2}+8 \alpha^{2} \theta\right)\right]\right. \\
& \left.-\left[\frac{4\left(\alpha^{2}+\theta\right)\left(2 \alpha^{2}+\theta\right)-\beta\left(3 \alpha^{4}+2 \theta^{2}+6 \alpha^{2} \theta\right)}{2\left(\alpha^{2}+\theta\right)}\right]^{2}\right\} \text {, } \\
& \mathrm{ID}=\frac{\alpha}{\theta}\left[\frac{8\left(\alpha^{2}+\theta\right)\left(3 \alpha^{2}+\theta\right)-3 \beta\left(5 \alpha^{4}+2 \theta^{2}+8 \alpha^{2} \theta\right)}{4\left(\alpha^{2}+\theta\right)\left(2 \alpha^{2}+\theta\right)-\beta\left(3 \alpha^{4}+2 \theta^{2}+6 \alpha^{2} \theta\right)}-\frac{4\left(\alpha^{2}+\theta\right)\left(2 \alpha^{2}+\theta\right)-\beta\left(3 \alpha^{4}+2 \theta^{2}+6 \alpha^{2} \theta\right)}{4\left(\alpha^{2}+\theta\right)^{2}}\right], \\
& \mathrm{Sk}=\frac{\alpha^{3}}{\theta^{3}\left(\alpha^{2}+\theta\right)^{2} \sigma_{\mathrm{X}}^{3}}\left\{\left[6\left(\alpha^{2}+\theta\right)\left(4 \alpha^{2}+\theta\right)-\frac{3 \beta}{4}\left(25 \alpha^{4}+7 \theta^{2}+35 \alpha^{2} \theta\right)\right]-\frac{3}{\left(\alpha^{2}+\theta\right)^{2}}\left[2\left(\alpha^{2}+\theta\right)\right.\right. \\
& \left.\times\left(3 \alpha^{2}+\theta\right)-\frac{3 \beta}{4}\left(5 \alpha^{4}+2 \theta^{2}+8 \alpha^{2} \theta\right)\right]\left[\left(\alpha^{2}+\theta\right)\left(2 \alpha^{2}+\theta\right)-\frac{\beta}{4}\left(3 \alpha^{4}+2 \theta^{2}+6 \alpha^{2} \theta\right)\right] \\
& \left.+\frac{2}{\left(\alpha^{2}+\theta\right)^{4}}\left[\left(\alpha^{2}+\theta\right)\left(2 \alpha^{2}+\theta\right)-\frac{\beta}{4}\left(3 \alpha^{4}+2 \theta^{2}+6 \alpha^{2} \theta\right)\right]^{3}\right\} \text {, } \\
& \mathrm{Ku}=\frac{\alpha^{4}}{\theta^{4}\left(\alpha^{2}+\theta\right)^{2} \sigma_{\mathrm{X}}^{4}}\left\{\left[24\left(\alpha^{2}+\theta\right)\left(5 \alpha^{2}+\theta\right)-\frac{30 \beta}{4}\left(14 \alpha^{4}+3 \theta^{2}+18 \alpha^{2} \theta\right)\right]-\frac{4}{\left(\alpha^{2}+\theta\right)^{2}}\left[6\left(\alpha^{2}+\theta\right)\right.\right. \\
& \left.\times\left(4 \alpha^{2}+\theta\right)-\frac{3 \beta}{4}\left(25 \alpha^{4}+7 \theta^{2}+35 \alpha^{2} \theta\right)\right]\left[\left(\alpha^{2}+\theta\right)\left(2 \alpha^{2}+\theta\right)-\frac{\beta}{4}\left(3 \alpha^{4}+2 \theta^{2}+6 \alpha^{2} \theta\right)\right] \\
& +\frac{6}{\left(\alpha^{2}+\theta\right)^{4}}\left[\left(\alpha^{2}+\theta\right)\left(6 \alpha^{2}+2 \theta\right)-\frac{3 \beta}{4}\left(5 \alpha^{4}+2 \theta^{2}+8 \alpha^{2} \theta\right)\right]\left[\left(\alpha^{2}+\theta\right)\left(2 \alpha^{2}+\theta\right)\right. \\
& \left.\left.-\frac{\beta}{4}\left(3 \alpha^{4}+2 \theta^{2}+6 \alpha^{2} \theta\right)\right]^{2}-\frac{3}{\left(\alpha^{2}+\theta\right)^{6}}\left[\left(\alpha^{2}+\theta\right)\left(2 \alpha^{2}+\theta\right)-\frac{\beta}{4}\left(3 \alpha^{4}+2 \theta^{2}+6 \alpha^{2} \theta\right)\right]^{4}\right\},
\end{aligned}
$$

where $\sigma_{X}=\sqrt{V(X)}$ is the standard deviation of $X$. Table 1 presents some values of these statistics for various parameters as in Figure 1.

Table 1 Statistics for the PTJ distribution with some various values of parameters $\alpha, \theta$ and $\beta$.

\begin{tabular}{ccccccccc}
\hline Figure & $\alpha$ & $\theta$ & $\beta$ & $\mathrm{E}(\mathrm{X})$ & $\mathrm{V}(\mathrm{X})$ & $\mathrm{ID}$ & $\mathrm{Sk}$ & $\mathrm{Ku}$ \\
\hline $\mathrm{a}$ & 0.05 & 0.05 & 0.75 & 0.66 & 0.53 & 0.81 & 2.69 & 15.03 \\
$\mathrm{~b}$ & 0.15 & 0.15 & 0.50 & 0.85 & 0.86 & 1.01 & 2.39 & 11.80 \\
$\mathrm{c}$ & 1.50 & 0.50 & 0.20 & 5.01 & 16.14 & 3.22 & 1.56 & 6.66 \\
$\mathrm{~d}$ & 3.00 & 0.50 & 0.70 & 8.54 & 43.08 & 5.05 & 1.80 & 8.50 \\
\hline
\end{tabular}

Proposition 2 If $\mathrm{X} \sim \operatorname{PTJ}(\alpha, \theta, \beta)$ then the moment generating function (mgf) of $\mathrm{X}$ is;

$$
\begin{aligned}
& \mathrm{M}_{\mathrm{X}}(\mathrm{t})=\left(\frac{\theta}{\alpha^{2}+\theta}\right)^{2}\left\{\frac { 2 \beta } { 2 \theta - \alpha ( \mathrm { e } ^ { \mathrm { t } } - 1 ) } \left[\frac{\alpha^{2} \theta}{2 \theta-\alpha\left(\mathrm{e}^{\mathrm{t}}-1\right)}\left(1+\frac{2 \alpha^{2}}{2 \theta-\alpha\left(\mathrm{e}^{\mathrm{t}}-1\right)}\right)\right.\right. \\
&\left.\left.+\left(\alpha^{2}+\theta\right)\left(1+\frac{\alpha^{2}}{2 \theta-\alpha\left(\mathrm{e}^{\mathrm{t}}-1\right)}\right)\right]-\frac{(\beta-1)\left(\alpha^{2}+\theta\right)}{\theta-\alpha\left(\mathrm{e}^{\mathrm{t}}-1\right)}\left(1+\frac{\alpha^{2}}{\theta-\alpha\left(\mathrm{e}^{\mathrm{t}}-1\right)}\right)\right\}
\end{aligned}
$$


where $\mathrm{t}<\log \left(\frac{\theta}{\alpha}+1\right)$ and the parameters $\alpha, \theta>0$ and $-1 \leq \beta \leq 1$.

Proof The mgf of a mixed Poisson distribution can be obtained from;

$$
\begin{aligned}
& \mathrm{M}_{\mathrm{X}}(\mathrm{t})= \mathrm{E}\left[\mathrm{E}\left(\mathrm{e}^{\mathrm{tx}} \mid \lambda\right)\right]=\int_{0}^{\infty}\left[\sum_{\mathrm{x}=0}^{\infty} \frac{\mathrm{e}^{\mathrm{tx}} \mathrm{e}^{-\lambda} \lambda^{\mathrm{x}}}{\Gamma(\mathrm{x}+1)}\right] \mathrm{g}_{\mathrm{TJ}}(\lambda) \mathrm{d} \lambda, \\
& \text { since } \sum_{\mathrm{x}=0}^{\infty} \frac{\mathrm{e}^{\mathrm{tx}} \mathrm{e}^{-\lambda} \lambda^{\mathrm{x}}}{\Gamma(\mathrm{x}+1)}=\mathrm{e}^{\lambda\left(\mathrm{e}^{\mathrm{t}}-1\right)} \text { and } \mathrm{g}_{\mathrm{TJ}}(\lambda) \text { as in }(2) \text {, we obtain; } \\
& \mathrm{M}_{\mathrm{X}}(\mathrm{t})= \frac{\theta^{2}}{\alpha\left(\alpha^{2}+\theta\right)^{2}}\left\{2 \beta \int_{0}^{\infty}(\alpha \lambda+1)\left(\alpha^{2}+\theta+\alpha \theta \lambda\right) \mathrm{e}^{-\left[\frac{2 \theta}{\alpha}-\left(\mathrm{e}^{\mathrm{t}}-1\right)\right] \lambda} \mathrm{d} \lambda\right. \\
&\left.-(\beta-1)\left(\alpha^{2}+\theta\right) \int_{0}^{\infty}(\alpha \lambda+1) \mathrm{e}^{-\left[\frac{\theta}{\alpha}-\left(\mathrm{e}^{\mathrm{t}}-1\right)\right] \lambda} \mathrm{d} \lambda\right\}, \\
&= \frac{\theta^{2}}{\alpha\left(\alpha^{2}+\theta\right)^{2}}\left\{2 \beta\left[\frac{\alpha^{2} \Gamma(2) \alpha\left(\alpha^{2}+\theta\right)}{\left(2 \theta-\alpha\left(\mathrm{e}^{\mathrm{t}}-1\right)\right)^{2}}+\frac{\alpha^{3} \Gamma(3) \alpha^{2} \theta}{\left(2 \theta-\alpha\left(\mathrm{e}^{\mathrm{t}}-1\right)\right)^{3}}+\frac{\alpha \Gamma(1)\left(\alpha^{2}+\theta\right)}{2 \theta-\alpha\left(\mathrm{e}^{\mathrm{t}}-1\right)}+\frac{\alpha^{2} \Gamma(2) \alpha \theta}{\left(2 \theta-\alpha\left(\mathrm{e}^{\mathrm{t}}-1\right)\right)^{2}}\right]\right. \\
&\left.-(\beta-1)\left(\alpha^{2}+\theta\right)\left[\frac{\alpha^{2} \Gamma(2) \alpha}{\left(\theta-\alpha\left(\mathrm{e}^{\mathrm{t}}-1\right)\right)^{2}}+\frac{\alpha \Gamma(1)}{\theta-\alpha\left(\mathrm{e}^{\mathrm{t}}-1\right)}\right]\right\} .
\end{aligned}
$$

Finally, we obtain $\mathrm{M}_{\mathrm{X}}(\mathrm{t})$ in (16).

Proposition 3 If $\mathrm{X} \sim \operatorname{PTJ}(\alpha, \theta, \beta)$ then the probability generating function (pgf) of $\mathrm{X}$ is;

$$
\begin{aligned}
\mathrm{H}_{\mathrm{X}}(\mathrm{s}) & =\left(\frac{\theta}{\alpha^{2}+\theta}\right)^{2}\left\{\frac { 2 \beta } { 2 \theta + \alpha ( 1 - \mathrm { s } ) } \left[\frac{\alpha^{2} \theta}{2 \theta+\alpha(1-\mathrm{s})}\left(1+\frac{2 \alpha^{2}}{2 \theta+\alpha(1-\mathrm{s})}\right)\right.\right. \\
& \left.\left.+\left(\alpha^{2}+\theta\right)\left(1+\frac{\alpha^{2}}{2 \theta+\alpha(1-\mathrm{s})}\right)\right]-\frac{(\beta-1)\left(\alpha^{2}+\theta\right)}{\theta+\alpha(1-\mathrm{s})}\left(1+\frac{\alpha^{2}}{\theta+\alpha(1-\mathrm{s})}\right)\right\},
\end{aligned}
$$

where the parameters $\alpha, \theta>0$ and $-1 \leq \beta \leq 1$.

Proof The pgf of a mixed Poisson distribution can be obtained by utilizing the pgf of the Poisson distribution as follows;

$$
\begin{aligned}
& \mathrm{H}_{\mathrm{X}}(\mathrm{s})=\mathrm{E}\left[\mathrm{E}\left(\mathrm{s}^{\mathrm{x}} \mid \lambda\right)\right]=\int_{0}^{\infty}\left[\sum_{\mathrm{x}=0}^{\infty} \frac{\mathrm{s}^{\mathrm{x}} \mathrm{e}^{-\lambda} \lambda^{\mathrm{x}}}{\Gamma(\mathrm{x}+1)}\right] \mathrm{g}_{\mathrm{TJ}}(\lambda) \mathrm{d} \lambda, \\
& \text { since } \sum_{\mathrm{x}=0}^{\infty} \frac{\mathrm{s}^{\mathrm{x}} \mathrm{e}^{-\lambda} \lambda^{\mathrm{x}}}{\Gamma(\mathrm{x}+1)}=\mathrm{e}^{-(1-\mathrm{s}) \lambda} \text { and } \mathrm{g}_{\mathrm{TJ}}(\lambda) \text { as in (2), we have; }
\end{aligned}
$$




$$
\begin{aligned}
\mathrm{H}_{\mathrm{X}}(\mathrm{s})= & \frac{\alpha^{-1} \theta^{2}}{\left(\alpha^{2}+\theta\right)^{2}}\left\{2 \beta \int_{0}^{\infty}(\alpha \lambda+1)\left(\alpha^{2}+\theta+\alpha \theta \lambda\right) \mathrm{e}^{-\left[\frac{2 \theta}{\alpha}+(1-\mathrm{s})\right] \lambda} \mathrm{d} \lambda-(\beta-1)\left(\alpha^{2}+\theta\right) \int_{0}^{\infty}(\alpha \lambda+1) \mathrm{e}^{-\left[\frac{\theta}{\alpha}+(1-s)\right] \lambda} \mathrm{d} \lambda\right\} \\
= & \frac{\theta^{2}}{\alpha\left(\alpha^{2}+\theta\right)^{2}}\left\{2 \beta\left\{\frac{\alpha^{2} \Gamma(2) \alpha\left(\alpha^{2}+\theta\right)}{[2 \theta+\alpha(1-s)]^{2}}+\frac{\alpha^{3} \Gamma(3) \alpha^{2} \theta}{[2 \theta+\alpha(1-s)]^{3}}+\frac{\alpha \Gamma(1)\left(\alpha^{2}+\theta\right)}{2 \theta+\alpha(1-s)}+\frac{\alpha^{2} \Gamma(2) \alpha \theta}{[2 \theta+\alpha(1-s)]^{2}}\right\}\right. \\
& \left.-(\beta-1) \frac{\alpha \Gamma(1)\left(\alpha^{2}+\theta\right)}{\theta+\alpha(1-s)}\left[1+\frac{\alpha^{2}}{\theta+\alpha(1-s)}\right]\right\} .
\end{aligned}
$$

Finally, we obtain $\mathrm{H}_{\mathrm{X}}(\mathrm{s})$ in (17). Alternatively, the pgf of the PTJ distribution can be obtained by setting $s=e^{t}$ as the mgf of $X$ as in (16).

\section{Generating a random variable of the PTJ distribution}

Now we can generate a variate X from the PJT distribution with the parameters $\alpha, \theta$ and $\beta$ by the following algorithm.

\section{Algorithm 1}

Step 1 Generate $\lambda$ with the inverse cdf of the TJ distribution in (5) as follows;

1) Generate $U$ from the uniform on interval $[0,1]$.

2) Set $\mathrm{c}=\frac{\alpha^{2}+\theta}{\alpha^{2}}$ and $\lambda$ as follows:

$$
\lambda=\frac{\alpha}{\theta}\left\{-\mathrm{c}-\mathrm{W}_{-1}\left\{-\mathrm{ce}^{-\mathrm{c}}\left[1-\frac{1}{2 \beta}\left(1+\beta-\sqrt{(\beta+1)^{2}-4 \beta \mathrm{u}}\right)\right]\right\}\right. \text {. }
$$

Step 2 Generate $X$ from the $\operatorname{Pois}(\lambda)$.

\section{Parameter estimation of the PTJ distribution}

Parametric inference deals with the estimation of an unknown parameter of a chosen distribution. The experimenter assumes that $\tilde{\mathrm{x}}=\left(\mathrm{x}_{1}, \ldots, \mathrm{X}_{\mathrm{n}}\right)$ is a random sample $\left(\mathrm{X}_{1}, \ldots, \mathrm{X}_{\mathrm{n}}\right)$ such that $\mathrm{X}_{\mathrm{i}}$ are independent and identically distributed random variables when $X_{i} \sim \operatorname{PTJ}(\alpha, \theta, \beta)$. In this study, the method of moments and the maximum likelihood estimation are introduced to estimate parameters $\alpha, \theta$ and $\beta$.

\section{Method of moments}

The method of moment estimators (MMEs), i.e., $\hat{\alpha}, \hat{\theta}$ and $\hat{\beta}$ of parameters $\alpha, \theta$ and $\beta$, respectively can be obtained by solving the following equations, i.e., $\mathrm{m}_{\mathrm{k}}=\mu_{\mathrm{k}}^{\prime}$;

$$
\begin{aligned}
& \mathrm{m}_{1}=\frac{1}{\left(\alpha^{2}+\theta\right)^{2}}\left(\frac{\alpha}{\theta}\right)\left[\left(\alpha^{2}+\theta\right)\left(2 \alpha^{2}+\theta\right)-\frac{\beta}{4}\left(3 \alpha^{4}+2 \theta^{2}+6 \alpha^{2} \theta\right)\right], \\
& \mathrm{m}_{2}=\frac{1}{\left(\alpha^{2}+\theta\right)^{2}}\left(\frac{\alpha}{\theta}\right)^{2}\left[\left(\alpha^{2}+\theta\right)\left(6 \alpha^{2}+2 \theta\right)-\frac{\beta}{4}\left(15 \alpha^{4}+6 \theta^{2}+24 \alpha^{2} \theta\right)\right], \\
& \mathrm{m}_{3}=\frac{1}{\left(\alpha^{2}+\theta\right)^{2}}\left(\frac{\alpha}{\theta}\right)^{3}\left[\left(\alpha^{2}+\theta\right)\left(24 \alpha^{2}+6 \theta\right)-\frac{\beta}{4}\left(75 \alpha^{4}+21 \theta^{2}+105 \alpha^{2} \theta\right)\right],
\end{aligned}
$$

where $\mathrm{m}_{\mathrm{k}}$ is the sample moments, i.e., $\mathrm{m}_{\mathrm{k}}=\frac{1}{\mathrm{n}} \sum_{\mathrm{i}=1}^{\mathrm{n}} \mathrm{x}_{\mathrm{i}}^{\mathrm{k}}, \mathrm{k}=1,2,3$. 
Since Eqs. (18) and (19) are no closed-form expression thus we use a numerical method by using the gmm package in the R stats package [18] to solve a non-linear model [19].

\section{Maximum likelihood estimation}

Let $X_{1}, \ldots, X_{n}$ be a random observation of size $n$ from the PTJ distribution with the pmf in (4). The log-likelihood function for the parameter vector of $\tilde{\omega}=(\alpha, \theta, \beta)$ can be written as;

$$
\begin{aligned}
\log L(\tilde{\omega})= & 2 \mathrm{n} \log \theta-2 \mathrm{n} \log \left(\alpha^{2}+\theta\right)+\sum_{\mathrm{i}=1}^{\mathrm{n}} \log \alpha^{\mathrm{x}_{\mathrm{i}}}\left\{\frac { 2 \beta } { ( \alpha + 2 \theta ) ^ { \mathrm { x } _ { \mathrm { i } } + 1 } } \left[\frac{\alpha^{2} \theta\left(\mathrm{x}_{\mathrm{i}}+1\right)}{\alpha+2 \theta}\left(1+\frac{\alpha^{2}\left(\mathrm{x}_{\mathrm{i}}+2\right)}{\alpha+2 \theta}\right)\right.\right. \\
& \left.\left.+\left(\alpha^{2}+\theta\right)\left(1+\frac{\alpha^{2}\left(\mathrm{x}_{\mathrm{i}}+1\right)}{\alpha+2 \theta}\right)\right]-\frac{(\beta-1)\left(\alpha^{2}+\theta\right)}{(\alpha+\theta)^{\mathrm{x}_{\mathrm{i}}+1}}\left[1+\frac{\alpha^{2}\left(\mathrm{x}_{\mathrm{i}}+1\right)}{\alpha+\theta}\right]\right\} .
\end{aligned}
$$

To estimate the unknown parameters $\alpha, \theta$ and $\beta$, we take the partial derivatives with respect to $\alpha$, $\theta$ and $\beta$ and equate them to 0 , i.e.,

$\frac{\partial \log \mathrm{L}(\tilde{\omega})}{\partial \alpha}=0, \frac{\partial \log \mathrm{L}(\tilde{\omega})}{\partial \theta}=0, \frac{\partial \log \mathrm{L}(\tilde{\omega})}{\partial \beta}=0$.

Since the Eq. (21) cannot be derived in closed forms, thus we used the numerical method of the 3-dimensional Newton-Raphson type procedure. We obtain the solutions of the maximum likelihood estimators (MLEs) of $\alpha, \theta$ and $\beta$ from the Eq. (21) by using the nlm function in the R stats package [18].

\section{Simulation}

We run a simulation study for parameter estimation of the PTJ parameters. The simulations are described as:

1) The sample sizes are taken as $\mathrm{n}=20,40,60,80,100,150,300$ and 500 .

2) The data are generated from the $\operatorname{PTJ}(\alpha, \theta, \beta)$ where the true parameter values are set as 4 -cases as in Table 1.

3) Each situation is repeated $T=1000$ times.

4) Formulas used for calculating the bias and root mean square error (RMSE) values of $\hat{\alpha}, \hat{\theta}$ and $\hat{\beta}$ are given by ;

$$
\begin{aligned}
& \operatorname{Bias}(\hat{\alpha})=\frac{1}{\mathrm{~T}}\left(\hat{\alpha}_{\mathrm{t}}-\alpha\right), \operatorname{Bias}(\hat{\theta})=\frac{1}{\mathrm{~T}}\left(\hat{\theta}_{\mathrm{t}}-\theta\right), \operatorname{Bias}(\hat{\beta})=\frac{1}{\mathrm{~T}}\left(\hat{\beta}_{\mathrm{t}}-\beta\right), \\
& \operatorname{RMSE}(\hat{\alpha})=\sqrt{\frac{1}{\mathrm{~T}} \sum_{\mathrm{t}=1}^{\mathrm{T}}\left(\hat{\alpha}_{\mathrm{t}}-\alpha\right)^{2}}, \operatorname{RMSE}(\hat{\theta})=\sqrt{\frac{1}{\mathrm{~T}} \sum_{\mathrm{t}=1}^{\mathrm{T}}\left(\hat{\theta}_{\mathrm{t}}-\theta\right)^{2}}, \text { and } \operatorname{RMSE}(\hat{\beta})=\sqrt{\frac{1}{\mathrm{~T}} \sum_{\mathrm{t}=1}^{\mathrm{T}}\left(\hat{\beta}_{\mathrm{t}}-\beta\right)^{2}} .
\end{aligned}
$$

The results from simulated data sets are reported in Tables 2 - 5. The average bias and RMSE values of the MMEs and MLEs are decreased when the sample size increases for all cases. Tables 2 - 5 indicate that the MLEs have the estimated value close to the true parameters more than the MMEs in all cases. However, the results in Tables $\mathbf{2}$ and 3, data sets with under-dispersion and equi-dispersion, indicate that the MMEs give a poor estimated value, but the MLEs still give the estimated value close to the true parameters. 
Table 2 The bias and RMSE values of the MMEs and MLEs of the PTJ distribution with parameters $\alpha=0.05, \theta=0.05$ and $\beta=0.75$ (ID =0.81: Under-dispersion).

\begin{tabular}{cccccccc}
\hline & & & MMEs & & \multicolumn{3}{c}{ MLEs } \\
\hline $\mathrm{n}$ & & $\alpha$ & $\theta$ & $\beta$ & $\alpha$ & $\theta$ & $\beta$ \\
\hline 20 & Bias & 0.9373 & 2.3346 & -0.5100 & 0.0533 & 0.0449 & -0.2052 \\
\hline & RMSE & 2.3341 & 6.2416 & 0.6289 & 0.1772 & 0.1593 & 0.3783 \\
\hline 40 & Bias & 0.8306 & 2.1763 & -0.5058 & 0.0506 & 0.0457 & -0.1312 \\
\hline 60 & RMSE & 1.9283 & 5.5675 & 0.6523 & 0.1148 & 0.1064 & 0.2450 \\
\hline & Bias & 0.7692 & 2.0095 & -0.5284 & 0.0311 & 0.0295 & -0.1252 \\
\hline 80 & RMSE & 1.7479 & 4.9456 & 0.6768 & 0.0927 & 0.0862 & 0.2216 \\
\hline & Bias & 0.6787 & 1.7916 & -0.4955 & 0.0215 & 0.0182 & -0.1001 \\
\hline 100 & RMSE & 1.5221 & 4.5015 & 0.6495 & 0.0731 & 0.0688 & 0.1792 \\
\hline & RMSE & 0.6331 & 1.6497 & -0.4693 & 0.0097 & 0.0066 & -0.0851 \\
\hline 150 & Bias & 1.4628 & 4.2898 & 0.6318 & 0.0553 & 0.0547 & 0.1520 \\
\hline 300 & RMSE & 1.0630 & 2.8360 & 0.6439 & 0.0441 & 0.0425 & 0.1259 \\
\hline & Bias & 0.3315 & 0.6865 & -0.4269 & 0.0044 & -0.0052 & -0.0335 \\
\hline 500 & RMSE & 0.6594 & 1.4879 & 0.5845 & 0.0262 & 0.0235 & 0.0682 \\
\hline & Bias & 0.1995 & 0.5269 & -0.4171 & 0.0040 & -0.0005 & -0.0080 \\
\hline & RMSE & 0.4052 & 0.9885 & 0.5529 & 0.0235 & 0.0191 & 0.0370 \\
\hline
\end{tabular}

Table 3 The bias and RMSE values of the MMEs and MLEs of the PTJ distribution with parameters $\alpha=0.15, \theta=0.15$ and $\beta=0.5$ ( ID =1.01: equi-dispersion).

\begin{tabular}{|c|c|c|c|c|c|c|c|}
\hline \multirow[b]{2}{*}{$\mathrm{n}$} & & \multicolumn{3}{|c|}{ MMEs } & \multicolumn{3}{|c|}{ MLES } \\
\hline & & $\alpha$ & $\theta$ & $\beta$ & $\alpha$ & $\theta$ & $\beta$ \\
\hline \multirow[t]{2}{*}{20} & Bias & 1.0827 & 2.4119 & -0.3092 & 0.1021 & 0.1108 & -0.1012 \\
\hline & RMSE & 2.9373 & 6.4841 & 0.4890 & 0.7697 & 0.7872 & 0.3399 \\
\hline \multirow[t]{2}{*}{40} & Bias & 1.0539 & 2.3359 & -0.2828 & 0.0548 & 0.0481 & -0.0101 \\
\hline & RMSE & 2.8191 & 6.1521 & 0.5045 & 0.4617 & 0.4658 & 0.2607 \\
\hline \multirow[t]{2}{*}{60} & Bias & 0.7614 & 1.6701 & -0.2905 & 0.0507 & 0.0427 & 0.0114 \\
\hline & RMSE & 1.9507 & 4.4498 & 0.5031 & 0.3992 & 0.4309 & 0.2248 \\
\hline \multirow[t]{2}{*}{80} & Bias & 0.5905 & 1.2806 & -0.2398 & 0.0421 & 0.0350 & 0.0599 \\
\hline & RMSE & 1.7703 & 3.8296 & 0.4734 & 0.3713 & 0.4067 & 0.2133 \\
\hline \multirow[t]{2}{*}{100} & Bias & 0.6062 & 1.2481 & -0.1811 & 0.0371 & 0.0261 & 0.0728 \\
\hline & RMSE & 1.7036 & 3.7201 & 0.4306 & 0.3510 & 0.3276 & 0.2112 \\
\hline \multirow[t]{2}{*}{150} & Bias & 0.4756 & 0.8735 & -0.1464 & 0.0289 & 0.0099 & 0.0753 \\
\hline & RMSE & 1.5260 & 2.6292 & 0.3851 & 0.3190 & 0.2868 & 0.2127 \\
\hline \multirow[t]{2}{*}{300} & Bias & 0.2702 & 0.4416 & -0.0984 & 0.0078 & -0.0043 & 0.0421 \\
\hline & RMSE & 0.8611 & 1.2048 & 0.3404 & 0.2737 & 0.2512 & 0.1163 \\
\hline \multirow[t]{2}{*}{500} & Bias & 0.1392 & 0.2259 & -0.0346 & -0.0027 & -0.0062 & 0.0274 \\
\hline & RMSE & 0.4721 & 0.6915 & 0.2839 & 0.2301 & 0.2005 & 0.1180 \\
\hline
\end{tabular}


Table 4 The bias and RMSE values of the MMEs and MLEs of the PTJ distribution with parameters $\alpha=1.5, \theta=0.5$ and $\beta=0.2$ ( ID = 3.22: over-dispersion).

\begin{tabular}{cccccccc}
\hline & & \multicolumn{3}{c}{ MMEs } & \multicolumn{3}{c}{ MLEs } \\
\hline $\mathrm{n}$ & & $\alpha$ & $\theta$ & $\beta$ & $\alpha$ & $\beta$ \\
\hline 20 & Bias & 0.7590 & 0.3195 & -0.1082 & 0.1094 & 0.0532 & 0.0563 \\
\hline & RMSE & 5.4854 & 2.0761 & 0.4476 & 0.4951 & 0.2165 & 0.4536 \\
\hline 40 & Bias & 0.4788 & 0.2162 & -0.1659 & 0.0662 & 0.0332 & -0.0007 \\
\hline 60 & RMSE & 4.5362 & 1.6587 & 0.4815 & 0.4853 & 0.2108 & 0.4736 \\
\hline & Bias & 0.5930 & 0.2336 & -0.1270 & 0.0105 & -0.0081 & 0.0375 \\
\hline 80 & RMSE & 4.2059 & 1.5319 & 0.4692 & 0.4389 & 0.2031 & 0.4260 \\
\hline 100 & Bias & 0.5433 & 0.3195 & -0.1082 & 0.1094 & 0.0532 & 0.0563 \\
\hline & RMSE & 4.0495 & 2.0761 & 0.4476 & 0.4951 & 0.2165 & 0.4536 \\
\hline 150 & Bias & 0.4241 & 0.1364 & -0.1059 & 0.0367 & 0.0248 & 0.1248 \\
\hline 300 & Bias & 0.4946 & 0.1608 & -0.0813 & 0.0130 & 0.0209 & 0.1692 \\
\hline 500 & RMSE & 3.4496 & 1.1713 & 0.4578 & 0.4229 & 0.1686 & 0.3600 \\
\hline & Bias & 0.2993 & 0.0965 & -0.0589 & 0.0520 & 0.0049 & 0.0777 \\
\hline & RMSE & 2.5698 & 0.8905 & 0.4220 & 0.3629 & 0.0773 & 0.1770 \\
\hline & & 0.1705 & 0.0767 & -0.0469 & 0.0289 & -0.0295 & 0.0279 \\
\hline
\end{tabular}

Table 5 The bias and RMSE values of the MMEs and MLEs of the PTJ distribution with parameters $\alpha=3, \theta=0.5$ and $\beta=0.7$ ( ID = 5.05: over-dispersion).

\begin{tabular}{cccccccc}
\hline & & & MMEs & & & MLEs \\
\hline $\mathrm{n}$ & & $\alpha$ & $\theta$ & $\beta$ & $\alpha$ & $\theta$ & $\beta$ \\
\hline 20 & Bias & -0.3212 & -0.0049 & -0.3249 & 0.0872 & -0.0069 & -0.0332 \\
\hline & RMSE & 5.2880 & 1.0119 & 0.5170 & 0.8910 & 0.3195 & 0.1749 \\
\hline 40 & Bias & -0.7363 & 0.0756 & -0.3663 & 0.0261 & -0.0235 & -0.0165 \\
\hline & RMSE & 4.8868 & 0.9138 & 0.5416 & 0.8176 & 0.2513 & 0.1683 \\
\hline 60 & Bias & -0.4147 & -0.0203 & -0.3148 & 0.0846 & -0.0013 & -0.0334 \\
\hline 80 & RMSE & 4.6500 & 0.8671 & 0.5064 & 0.8007 & 0.2453 & 0.1609 \\
\hline & Bias & -0.2510 & -0.0001 & 0.2792 & 0.0461 & -0.0119 & -0.0323 \\
\hline 100 & RMSE & 4.5957 & 0.8636 & 0.4802 & 0.7943 & 0.2399 & 0.1416 \\
\hline 150 & Bias & -0.1103 & 0.0264 & -0.2237 & -0.0006 & -0.0161 & -0.0275 \\
\hline & RMSE & 4.5562 & 0.8592 & 0.4419 & 0.7371 & 0.2157 & 0.1237 \\
\hline 300 & Bias & 0.0557 & 0.0428 & -0.2057 & 0.2429 & 0.0159 & -0.0343 \\
\hline 500 & RMSE & 4.1688 & 0.7523 & 0.4302 & 0.6970 & 0.2014 & 0.1138 \\
\hline & Bias & 0.3991 & 0.0996 & -0.1158 & 0.0260 & -0.0113 & -0.0377 \\
\hline & RMSE & 3.7946 & 0.7114 & 0.3465 & 0.6038 & 0.1092 & 0.0768 \\
\hline & RMSE & 0.5631 & 0.1393 & -0.0956 & 0.0175 & -0.0089 & -0.0281 \\
\hline & 3.5453 & 0.7133 & 0.3243 & 0.4826 & 0.0819 & 0.0573 \\
\hline
\end{tabular}




\section{Application}

For an application study, some real data sets are fitting distributions such as the PTJ, PJ, PL and Pois distributions. The parameters of each distribution are estimated by the method of moments and the maximum likelihood estimation. The first data set (Data I) is the number of European red mites on apple leaves, which appear in [20]. The second data set (Data II) is the number of insects of Pyrausta nubilalis in 1937 [21]. The third data (Data III) is the number of hospitalizations, per family member, per year [22]. And the number of the outbreak of strikes (Data IV) in the south of London during World War II [23]. The summary of these data is shown in Table 6.

Table 6 Data summary of the real data sets.

\begin{tabular}{lcccccc}
\multicolumn{1}{c}{ Data } & $\mathbf{n}$ & Median & Mode & Mean & Variance & ID \\
\hline The number of European red mites per leaf & 150 & 1 & 0 & 1.1470 & 2.2736 & 1.9822 \\
The number of insects & 56 & 0 & 0 & 0.7500 & 1.3382 & 1.7843 \\
The number of hospitalizations per family member & 2,924 & 0 & 0 & 0.0985 & 0.1059 & 1.0751 \\
The number of outbreak of strikes & 156 & 1 & 1 & 0.9936 & 0.7419 & 0.7419 \\
\hline
\end{tabular}

In this study, the Kolmogorov-Smirnov (KS) test is used for the criteria for the goodness of fit test of each distribution, where the model has the smaller value of KS test that is the best fit for the data. The parameter estimates and the goodness of fit test for these data sets are illustrated in Tables 7 - 10. The results show that the maximum likelihood estimation gives the parameter estimates that make the fitting distributions close to the empirical data greater than the fitting distribution by using the parameter estimate of the method of moments. The fitted distribution with the parameters estimated by the maximum likelihood estimation gives the lower KS value than the fitted distribution with the parameters estimated by the method of moments in all cases (Tables 7 - 10). In Tables 7 and 8, the real data sets with over-dispersion, the PTJ distribution gives a lower value of the KS test compared to other distributions such as the PJ, PL and Poisson distributions. For count data with equi-dispersion and underdispersion, the Poisson distribution gives a lower KS value than other distributions (Tables 9 and 10). However, the fitted PTJ distribution with the parameters estimated by the maximum likelihood estimation gives the lower KS value than other distributions in count data with equi-dispersion (Table 9). We conclude that the PTJ distribution is appropriate to fit count data with over-dispersion. 
Table 7 Fitting distributions of the numbers of European red motes on apple leaves (X).

\begin{tabular}{|c|c|c|c|c|c|c|c|c|c|}
\hline \multirow{3}{*}{$\mathbf{X}$} & \multirow{3}{*}{$\begin{array}{c}\text { observe } \\
\text { d }\end{array}$} & \multicolumn{8}{|c|}{ Expected values of fitting distributions } \\
\hline & & \multicolumn{4}{|c|}{ Maximum likelihood estimation } & \multicolumn{4}{|c|}{ Method of moments } \\
\hline & & Pois & PL & PJ & PTJ & Pois & $\mathbf{P L}$ & $\mathbf{P J}$ & PTJ \\
\hline 0 & 70 & 47.65 & 67.26 & 68.91 & 69.01 & 47.66 & 57.89 & 64.5 & 66.26 \\
\hline 1 & 38 & 54.64 & 38.89 & 37.82 & 37.76 & 54.64 & 37.81 & 36.76 & 36.40 \\
\hline 2 & 17 & 31.33 & 21.24 & 20.42 & 20.37 & 31.33 & 23.18 & 20.96 & 20.32 \\
\hline 3 & 10 & 11.98 & 11.19 & 10.89 & 10.87 & 11.97 & 13.65 & 11.95 & 11.49 \\
\hline 4 & 9 & 3.43 & 5.74 & 5.75 & 5.74 & 3.43 & 7.82 & 6.81 & 6.56 \\
\hline 5 & 3 & 0.79 & 2.89 & 3.01 & 3.01 & 0.79 & 4.39 & 3.88 & 3.77 \\
\hline 6 & 2 & 0.15 & 1.43 & 1.56 & 1.57 & 0.15 & 2.42 & 2.21 & 2.18 \\
\hline 7 & 1 & 0.02 & 0.70 & 0.81 & 0.81 & 0.02 & 1.32 & 1.26 & 1.26 \\
\hline & $-\log L$ & 242.81 & 222.51 & 222.38 & 222.38 & - & - & - & - \\
\hline & AIC & 487.62 & 447.02 & 448.76 & 450.76 & - & - & - & - \\
\hline & BIC & 490.63 & 450.03 & 454.78 & 459.79 & - & - & - & - \\
\hline & $\hat{\lambda}$ & 1.1467 & - & - & - & 1.1466 & - & - & - \\
\hline & $\hat{\alpha}$ & - & - & 0.3552 & 0.4297 & - & - & 0.0033 & 0.0653 \\
\hline & $\hat{\theta}$ & - & 1.2602 & 0.3861 & 0.4674 & - & 1.0355 & 0.0025 & 0.0494 \\
\hline & $\hat{\beta}$ & - & - & - & 0.0580 & - & - & - & 0.1781 \\
\hline & $\mathrm{KS}$ test & 0.1490 & 0.0239 & 0.0202 & 0.0200 & 0.1490 & 0.0820 & 0.0449 & 0.0356 \\
\hline & $b$-value & $<0.0001$ & 0.8560 & 0.9154 & 0.9165 & $<0.0001$ & 0.5572 & 0.9265 & 0.9540 \\
\hline
\end{tabular}

Table 8 Fitting distributions of the numbers of insects (X).

\begin{tabular}{|c|c|c|c|c|c|c|c|c|c|}
\hline \multirow{3}{*}{$\mathbf{X}$} & \multirow{3}{*}{ Observed } & \multicolumn{8}{|c|}{ Expected values of fitting distributions } \\
\hline & & \multicolumn{4}{|c|}{ Maximum likelihood estimation } & \multicolumn{4}{|c|}{ Method of moments } \\
\hline & & Pois & PL & PJ & PTJ & Pois & PL & PJ & PTJ \\
\hline 0 & 33 & 26.45 & 31.52 & 31.98 & 32.19 & 25.16 & 21.61 & 28.21 & 30.22 \\
\hline 1 & 12 & 19.84 & 14.15 & 13.72 & 13.56 & 20.13 & 14.12 & 14.22 & 13.53 \\
\hline 2 & 6 & 7.44 & 6.08 & 5.88 & 5.80 & 8.05 & 8.65 & 7.03 & 6.25 \\
\hline 3 & 3 & 1.86 & 2.53 & 2.52 & 2.51 & 2.15 & 5.10 & 3.42 & 2.98 \\
\hline 4 & 1 & 0.35 & 1.03 & 1.08 & 1.09 & 0.43 & 2.92 & 1.65 & 1.46 \\
\hline 5 & 1 & 0.05 & 0.41 & 0.46 & 0.48 & 0.07 & 1.64 & 0.78 & 0.74 \\
\hline & $-\log L$ & 71.58 & 66.98 & 66.92 & 66.91 & - & - & - & - \\
\hline & AIC & 145.16 & 135.96 & 137.84 & 139.82 & - & - & - & - \\
\hline & $\mathrm{BIC}$ & 147.19 & 137.99 & 141.89 & 145.90 & - & - & - & - \\
\hline & $\hat{\lambda}$ & 0.7500 & - & - & - & 0.8000 & - & - & - \\
\hline & $\hat{\alpha}$ & - & & 0.0714 & 0.2886 & - & - & 0.4437 & 0.0831 \\
\hline & $\hat{\theta}$ & - & 1.8115 & 0.0998 & 0.4087 & - & 1.0355 & 0.5788 & 0.0711 \\
\hline & $\hat{\beta}$ & - & - & - & 0.1864 & - & - & - & 0.5887 \\
\hline & KS test & 0.1169 & 0.0265 & 0.0182 & 0.0145 & 0.1400 & 0.2034 & 0.0856 & 0.0496 \\
\hline & $p$-value & 0.4282 & 1.0000 & 1.0000 & 1.0000 & 0.2227 & 0.0195 & 0.8068 & 0.9991 \\
\hline
\end{tabular}


Table 9 Fitting distributions of the number of hospitalizations per family member (X).

\begin{tabular}{|c|c|c|c|c|c|c|c|c|c|}
\hline \multirow{3}{*}{$\mathbf{X}$} & \multirow{3}{*}{ Observed } & \multicolumn{8}{|c|}{ Expected value of fitting distributions } \\
\hline & & \multicolumn{4}{|c|}{ Maximum likelihood estimation } & \multicolumn{4}{|c|}{$\begin{array}{l}\text { Method of moments } \\
\end{array}$} \\
\hline & & Pois & PL & PJ & PTJ & Pois & PL & PJ & PTJ \\
\hline 0 & 2,659 & $2,649.72$ & $2,546.5$ & $2,661.75$ & $2,659.05$ & $2,649.72$ & $2,561.15$ & $2,562.07$ & $2,570.33$ \\
\hline 1 & 244 & 261.00 & 329.19 & 238.74 & 243.65 & 261.00 & 318.2 & 317.13 & 308.84 \\
\hline 2 & 19 & 12.85 & 42.17 & 21.41 & 19.67 & 12.85 & 39.2 & 39.25 & 38.98 \\
\hline $3+$ & 2 & 0.43 & 6.14 & 2.10 & 1.63 & 0.43 & 5.45 & 5.55 & 5.85 \\
\hline & $-\log \mathrm{L}$ & 972.26 & 993.62 & 969.25 & 969.07 & - & - & - & - \\
\hline & AIC & $1,946.52$ & $1,989.24$ & $1,942.50$ & $1,944.14$ & - & - & - & - \\
\hline & $\mathrm{BIC}$ & $1,952.50$ & $1,995.22$ & $1,954.46$ & $1,962.08$ & - & - & - & - \\
\hline & $\hat{\lambda}$ & 0.0985 & - & - & - & 0.0985 & - & - & - \\
\hline & $\hat{\alpha}$ & - & - & 0.1430 & 0.0785 & - & - & 0.0014 & 0.1333 \\
\hline & $\hat{\theta}$ & - & 7.5459 & 1.4713 & 1.0022 & - & 7.8649 & 0.0101 & 0.8667 \\
\hline & $\hat{\beta}$ & - & - & - & -0.4998 & - & - & - & 0.2333 \\
\hline & KS test & 0.0032 & 0.0385 & 0.0009 & 0.0001 & 0.0032 & 0.0335 & 0.0311 & 0.0303 \\
\hline & $p$-value & 1.0000 & 0.0003 & 1.0000 & 1.0000 & 1.0000 & 0.0029 & 0.0069 & 0.0092 \\
\hline
\end{tabular}

Table 10 Fitting distributions of the number of outbreak of strikes (X).

\begin{tabular}{|c|c|c|c|c|c|c|c|c|c|}
\hline \multirow{3}{*}{$\mathbf{X}$} & \multirow{3}{*}{ Observed } & \multicolumn{8}{|c|}{ Expected value of fitting distributions } \\
\hline & & \multicolumn{4}{|c|}{ MLEs } & \multicolumn{4}{|c|}{ MEs } \\
\hline & & Pois & PL & PJ & PTJ & Pois & PL & PJ & PTJ \\
\hline 0 & 46 & 57.76 & 75.24 & 56.57 & 70.00 & 57.76 & 77.80 & 79.14 & 69.72 \\
\hline 1 & 76 & 57.39 & 40.55 & 45.00 & 42.74 & 57.39 & 40.48 & 39.60 & 44.18 \\
\hline 2 & 24 & 28.51 & 20.73 & 26.86 & 22.60 & 28.51 & 20.01 & 19.43 & 23.04 \\
\hline 3 & 9 & 9.44 & 10.23 & 14.25 & 11.12 & 9.44 & 9.56 & 9.39 & 10.84 \\
\hline 4 & 4 & 2.35 & 4.93 & 7.09 & 5.24 & 2.35 & 4.46 & 4.48 & 4.79 \\
\hline \multirow[t]{2}{*}{$5+$} & 0 & 0.55 & 4.32 & 6.23 & 4.30 & 0.55 & 3.69 & 3.96 & 3.43 \\
\hline & $-\log \mathrm{L}$ & 191.94 & 212.36 & 208.00 & 208.79 & - & - & - & - \\
\hline & AIC & 385.88 & 426.72 & 420.00 & 423.58 & - & - & - & - \\
\hline & BIC & 388.93 & 429.77 & 426.10 & 432.73 & - & - & - & - \\
\hline & $\hat{\lambda}$ & 0.9936 & - & - & - & 0.9936 & - & - & - \\
\hline & $\hat{\alpha}$ & - & - & $4,878.70$ & 3.7682 & - & - & 0.4512 & 0.9111 \\
\hline & $\hat{\theta}$ & - & 1.4010 & $7,382.48$ & 5.8566 & - & 1.4751 & 0.5972 & 1.6556 \\
\hline & $\hat{\beta}$ & - & - & - & 0.0586 & - & - & - & -0.6296 \\
\hline & KS test & 0.0754 & 0.1874 & 0.1310 & 0.1538 & 0.0754 & 0.2038 & 0.2124 & 0.1782 \\
\hline & $p$-value & 0.3382 & $<0.0001$ & 0.0095 & 0.0012 & 0.3382 & $<0.0001$ & $<0.0001$ & 0.0001 \\
\hline
\end{tabular}




\section{Conclusions}

A new 3-parameter discrete distribution for modeling count data, so-called the Poisson transmuted Janardan (PTJ) distribution, is proposed, which is obtained by mixing the Poisson distribution and the transmuted Janardan distribution. The PTJ has 2 special models like the Poisson Janardan distribution by [8] and Poisson Lindley distribution by [4]. Some mathematical characteristics of the proposed distribution, including the moments, moment generating function and probability generating function, are introduced. We discuss the parameter estimation by using the moments of method and the maximum likelihood estimation and testing of hypotheses for the model parameters with the KS test. The simulation results indicate that the maximum likelihood estimation gives the estimated parameters close to the true parameters more than the method of moments. The results of the application study found that the PTJ distribution is appropriate to fit count data with over-dispersion. The practical relevance and applicability of the new model are demonstrated in 4 real data sets. We verify that some sub-models could provide similar fits while the PTJ distribution being more parsimonious models for count data with overdispersion.

\section{Acknowledgements}

The authors acknowledge profound thanks to anonymous referee for giving comments which have immensely improved the presentation of the paper.

\section{References}

[1] D Bhati, P Kumawat and E Gómez-Déniz. A new count model generated from mixed Poisson transmuted exponential family with an application to health care data. Comm. Stat. Theor. Meth. 2017; 46, 11060-76.

[2] D Karlis and E Xekalaki. Mixed Poisson distributions. Int. Stat. Rev. 2005; 73, 35-58.

[3] M Greenwood and GU Yule. An inquiry into the nature of frequency distributions representative of multiple happenings with particular reference to the occurrence of multiple attacks of disease or of repeated accidents. J. Roy. Stat. Soc. 1920; 83, 255-79.

[4] M Sankaran. The discrete Poisson-lindley distribution. Biometrics 1970; 26, 145-9.

[5] HS Sichel. On a distribution law for word frequencies. J. Am. Stat. Assoc. 1975; 70, 542-7.

[6] GE Willmot. On recursive evaluation of mixed Poisson probabilities and related quantities. Scand. Actuarial J. 1993; 18, 114-33.

[7] SA Al-Awadhi and ME Ghitany. Statistical properties of Poisson Lomax distribution and its application to repeated accident data. J. Appl. Stat. Sci. 2001; 10, 365-72.

[8] E Mahmoudi and H Zakerzadeh. Generalized Poisson-Lindley distribution. Comm. Stat. Theor. Meth. 2010; 39, 1785-98.

[9] H Zamani, N Ismail and P Faroughi. Poisson-weighted exponential univariate version and regression model with applications. J. Math. Stat. 2014; 10, 148-54.

[10] R Shanker, S Sharma, U Shanker, R Shanker and TA Leonida. The discrete Poisson-Janardan distribution with applications. Int. J. Soft Comput. Eng. 2014; 4, 31-3.

[11] E Gómez-Déniz and E Calderín-Ojeda. Parameters estimation for a new generalized geometric distribution. Comm. Stat. Simulat. Comput. 2015; 44, 2023-39.

[12] W Wongrin and W Bodhisuwan. The Poisson-generalised Lindley distribution and its applications. Songklanakarin J. Sci. Tech. 2016; 38, 645-56.

[13] AI Al-Omari, AM Al-khazaleh and LM Alzoubi. Transmuted Janardan distribution: A generalization of the Janardan distribution. J. Stat. Appl. Probab. 2017; 5, 1-11.

[14] R Shanker, S Sharma, U Shanker and R Shanker. Janardan distribution and its application to waiting times data. Indian J. Appl. Res. 2013; 3, 500-2.

[15] D Veberič. Lambert W function for applications in physics. Comput. Phys. Comm. 2012; 183, 26228.

[16] RM Corless, GH Gonnet, DE Hare, DJ Jeffrey and DE Knuth. On the Lambert W function. Adv. Comput. Math. 1996; 5, 329-59.

[17] WT Shaw and IR Buckley. The alchemy of probability distributions: Beyond Gram Charlier expansions, and a skew-kurtotic-normal distribution from a rank transmutation map, Available at: https://arxiv.org/abs/0901.043, accessed January 2020.

[18] R Core Team. R: A language and environment for statistical computing. R Foundation for Statistical Computing, Vienna, Austria, 2018. 
[19] P Chaussé. Computing generalized method of moments and generalized empirical likelihood with R. J. Stat. Software 2010; 34, 1-35.

[20] CI Bliss and RA Fisher. Fitting the negative binomial distribution to biological data. Biometrics 1953; 9, 176-200.

[21] G Beall. The fit and significance of contagious distributions when applied to observations on larval insects. Ecology 1940; 21, 460-74.

[22] SA Klugman, HH Panjer and GE Willmot. Loss models: From data to decisions. John Wiley \& Sons, New York, 1998.

[23] MH Alamatsaz, S Dey, T Dey and SS Harandi. Discrete generalized Rayleigh distribution. Pak. J. Stat. 2016; 32, 1-20. 